140 SOCIODEMOGRAPHIC AND HEALTH-RELATED FACTORS ASSOCIATED WITH TEMPORARY WORK DISABILITY IN A LARGE GERMAN CHEMICAL COMPANY: RESULTS OF A CROSS-SECTIONAL STUDY

M Claus*, M Schuster, S Webendörfer, C Oberlinner. Corporate Health Management, BASF SE, Ludwigshafen, Germany

\subsection{6/oemed-2018-ICOHabstracts.1538}

Introduction With the present study, we aimed to determine the occurrence of temporary work disability within 12 months in a large German chemical company. Furthermore, we assessed the association of sociodemographic and health-related factors with work disability.

Methods We used cross-sectional data, surveyed in occupational health checks-ups between January 2011 and December 2014 at the Ludwigshafen site (Germany). A blood sample, physical examination, anamnesis by a physician and a written questionnaire were part of the health check-up. Work disability in the year prior to participation was assessed using a single (categorical) item from the Work-Ability-Index. We used partial proportional odds models for ordinal response variables to assess the association of sociodemographic and healthrelated factors with work disability.

Results Altogether, 17351 employees participated in the voluntary health check-up. Excluding 386 persons with missing information and trainees, a final sample of 16965 persons was yielded. Respondents were on average 43.7 (SD: 9.7) years old and predominantly male $(79.0 \%)$. About one third $(32.8 \%)$ did not miss a single day, $40.8 \%$ up to nine days, $18.5 \%$ 10-24 days, 6.8\% 25-99 days, and 1.1\% 100 days or more. The proportion of respondents being unable to work for $\geq 10$ days in the last 12 months was comparatively high for older persons $(31.8 \%$; 50+years) vs younger persons $(22.6 \%$; <30 years), manual workers $(40.1 \%)$ vs managerial staff $(8.3 \%)$, rotating shift workers $(38.9 \%)$ vs day workers $(21.9 \%)$, obese $(38.1 \%)$ vs non-obese people $(19.6 \%)$, and smokers (35.3\%) vs non-smokers (20.6\%). In multivariable analyses, missing $\geq 10$ days was significantly more likely for older respondents, females, manual workers and skilled/supervisory workers (vs. managerial staff), rotating shift workers, obese people, and for smokers and former smokers (vs. nonsmokers).

Conclusion The findings of the present study could be considered a starting point for the implementation of targeted preventive measures to reduce work disability.

\section{SICK LEAVE ASSESSMENTS OF PEOPLE WITH PERSISTENT 'MEDICALLY UNEXPLAINED' PHYSICAL SYMPTOMS: A CROSS-SECTIONAL STUDY AMONG TWO GROUPS OF PHYSICIANS}

\footnotetext{
1,2,3 KHN Weerdesteijn, 1,2 FG Schaafsma* ${ }^{1,2} \mathrm{AJ}$ van der Beek, ${ }^{1,2} \mathrm{JR}$ Anema. 'Department of Public and Occupational Health within Public Health research institute, Amsterdam, The Netherlands; ${ }^{2}$ Research Centre for Insurance Medicine (KCVG), AMC-UMCG-UWV-VUmC, Amsterdam, The Netherlands; ${ }^{3}$ Department of Social Medical Affairs (SMZ), Dutch Social Security Agency (UWV), Amsterdam, the Netherlands
}

\subsection{6/oemed-2018-ICOHabstracts. 1539}

Introduction Sick leave assessments of people with persistent 'medically unexplained' physical symptoms (PPS) can be diffi- cult for physicians, and may show a variation in outcome.

This variation can be a result of a different working context or medical background of the assessor, or due to the objective of the assessment. To obtain more insight in the extent and reasons for assessment differences related to this group of patients we explored sick leave assessments of physicians working in the same health care field, with similar tasks, but with different objectives for the assessments.

Methods A cross sectional study was conducted among 50 occupational health physicians (OPs) and 43 insurance physicians (IPs) from the Netherlands. They attended a full day training on PPS, and were asked to assess the sick leave of nine video case vignettes of patients with PPS. Data were analysed using multinomial regression approach in SPSS.

Results Sick leave was less likely to be advised by IPs compared to OPs (OR $0.74,95 \% \mathrm{CI}$ : 0.56 to 0.97 ). For all physicians the sick leave assessment was influenced by their opinion on perceived limited work-related functioning, the cause of the health complaints, the diagnosis, and treatment options. For OPs, private life issues influencing the cause of the health complaints was taken into account more than for IPs.

Conclusion Although, the differences in the sick leave assessment were small among these physicians it seems that the objective of the assessment is of influence on the outcome. OPs may be more focussed on sick leave guidance and vocational rehabilitation options, whereas IPs may have a stricter objective with their assessments focussing on the certification of a sickness benefit.

\section{A TIME FOR REFLECTION: INTERNATIONAL WORK DISABILITY POLICY CHALLENGES AND DIRECTIONS}

${ }^{1} \mathrm{E}$ MacEachen, ${ }^{2} \mathrm{~K}$ Ekberg. ' University of Waterloo, Waterloo, Canada; ${ }^{2}$ Linkoping University, Linkoping, Sweden

\subsection{6/oemed-2018-ICOHabstracts. 1540}

Introduction Since the 1990's, there has been a move across international jurisdictions to policies and programs that focus on work ability, not disability, and on strategies that encourage employment integration of people with temporary and permanent impairments. Almost 30 years later, we are at a point where we can reflect on those programs: their ideals, what worked, and what did not work and why.

Method Top work disability researchers from 14 jurisdictions assembled in Toronto in 2017 to share knowledge about work disability conditions, policies and practical social, economic and political realities of work integration. Each addressed work disability policies in their country, current practices, and avenues for change. Issues raised were compared and contrasted and then summarised in using thematic analysis approaches.

Results Across jurisdictions, rising levels of disability claimants were a concern, together with increasing work absence due to mental illness, work disability in ageing populations, and limited social protection for self-employed workers. Many systems have been redesigned in recent years to address these and other issues using strategies including program coordination, tightened timelines, eligibility restrictions, and reduced income and health care support for sick listed workers. Employer 
activation, management of non-severe impairment, and when to begin work activation were locations of developing and disparate policies.

Conclusion A relationship between reduced number of people on disability benefits and increased employment is not always clear. More tracking is needed of employment outcomes for people who receive or are declined support. Strategies for successfully engaging employers and health care providers in work integration are still developing. Areas of emergent concern are effects of quality of the claimant encounter with providers and approaches to support the growing self-employment economy.

\section{DEVELOPMENT OF AN INTERVENTION TO ENHANCE SELF-MANAGEMENT AT WORK FOR WORKERS WITH A CHRONIC DISEASE USING INTERVENTION MAPPING}

AR Bosma*, CRL Boot, FG Schaafsma, JR Anema. VU University Medical Centre, Amsterdam Public Health research institute, department of Public and Occupational Health, Amsterdam, The Netherlands

\subsection{6/oemed-2018-ICOHabstracts. 1541}

Introduction The number of people in the working population diagnosed with one or more chronic diseases will increase. Self-management at work may facilitate workers with a chronic disease to continue working. Occupational and insurance physicians can play a key role in supporting this selfmanagement at work, as this fits within their preventive task. The aim of this project is to explore how occupational and insurance physicians can optimally support chronically ill workers in improving their self-management at work. We will develop an intervention that will fit within the needs of all stakeholders involved, using Intervention Mapping (IM) as a tool.

Methods The intervention will be developed with the use of the 6 step IM protocol:

- Needs assessment;

- Definition of change objectives;

- Selection of theory based methods and practical strategies;

- Program development;

- Adoption and implementation plan;

- Evaluation plan.

Step 1, the needs assessment will consist of a systematic review of the literature and a qualitative explorative study using focus groups. In the focus groups with chronically ill workers, occupational and insurance physicians we will explore their perceptions on self-management at work, their needs for support in improving self-management at work and the views of occupational and insurance physicians on providing this support.

Result The results of the needs assessment are expected early 2018 and will be available for presentation at the conference. Discussion The iterative steps of the IM protocol help to develop an intervention for occupational and insurance physicians that enables them to support workers with a chronic disease in improving self-management at work, to maintain productivity and to prevent sick leave and job loss.

\section{4 PERSON-RELATED FACTORS ASSOCIATED WITH WORK PARTICIPATION IN EMPLOYEES WITH HEALTH PROBLEMS: A SYSTEMATIC REVIEW}

M de Wit*, H Wind, CTJ Hulshof, MHW Frings-Dresen. Academic Medical Centre, department Coronel Institute of Occupational Health, Amsterdam Public Health research institute, Amsterdam, The Netherlands

\subsection{6/oemed-2018-ICOHabstracts. 1542}

Introduction Sick leave or work disability, is a major health problem, with negative consequences for the employee, employer and society. In order to prevent or decrease the duration of sick leave, it is important to know which factors influence work participation. Research revealed that besides disease-related factors, person-related factors like cognitions and perceptions of employees play a role in work retention and return to work after sick leave. Structured information about these factors can help occupational- and insurance physicians in their activities to decrease the duration of sick leave. However, structured information about the influence of person-related factors on work participation of workers with health problems is not available yet. This study aims to provide systematically assessed information about the association between person-related factors and work participation.

Methods A systematic review was conducted to explore the association between person-related factors and work participation. PubMed and PsycINFO were used to search for papers published between January 2007 and February 2017. There were no restrictions in study design. The methodological quality of included studies was assessed using quality assessment tools of the Joanna Briggs Institute.

Result 116 of 3465 studies were included which addressed the association between person-related factors and work participation. Evidence was found for a positive association between motivation, positive recovery expectations, self-efficacy, intern locus of control, perceived health, and optimism and work retention or return to work after sick leave. The personrelated factors catastrophizing, fear-avoidance coping, and perceiving work as a cause of the disability were negatively associated with work retention or return to work after sick leave. Discussion The results provide input for developing tailored interventions and provide guidance for occupational- and insurance physicians to facilitate returning to work and work retention. Further research is required to determine how these physicians could acquire and apply information about person-related factors.

\section{CHARACTERISTICS OF INDIVIDUALS RECEIVING DISABILITY BENEFITS IN THE NETHERLANDS AND PREDICTORS OF LEAVING THE BENEFIT SCHEME. A FIVE-YEAR FOLLOW-UP STUDY} ${ }^{1,2,3}$ I Louwerse*, ${ }^{1,3} \mathrm{MA}$ Huysmans, ${ }^{1,2} \mathrm{HJ}$ van Rijssen, ${ }^{1,3} \mathrm{AJ}$ van der Beek, ${ }^{1,3} \mathrm{JR}$ Anema.
${ }^{1}$ Department of Public and Occupational health, VU University Medical Centre, Amsterdam,
The Netherlands; ${ }^{2}$ Dutch Institute of Employee Benefit Schemes (UWV), Amsterdam, The
Netherlands; ${ }^{3}$ Research Centre for Insurance Medicine, AMC-UMCG-VUmC, Amsterdam,
The Netherlands

\subsection{6/oemed-2018-ICOHabstracts. 1543}

Introduction Nowadays, work disability is one of the biggest social and labour market challenges for policy makers in 